\title{
Metamorfosis del TURISMo Y TRANSFORMACIONES URBANAS: HACIA EL DESARROLLO DE UN MODELO DE TURISMO SOSTENIBLE ${ }^{1}$
}

Tourism metamorphosis and urban transformations: towards the development of a sustainable tourism model

\author{
Marina Haro Aragú \\ Instituto Universitario de Investigación, de Inteligencia e Innovación Turís- \\ tica de la Universidad de Málaga (i3t) \\ marinaharo94@gmail.com \\ https://orcid.org/0000-0001-9988-5459

\section{Josefa García Mestanza} \\ Instituto Universitario de Investigación, de Inteligencia e Innovación Turís- \\ tica de la Universidad de Málaga (i3t) \\ jgm@uma.es \\ https://orcid.org/0000-0002-3865-3061
}

1. La investigación que da lugar a este artículo es parte del resultado del proyecto de investigación titulado "Saturación turística en destinos costeros españoles. Estrategias de decrecimiento turístico. Una aproximación desde la dimensión social” (RTI2018094844-B-C33) del Ministerio de Ciencia, Innovación y Universidades (Plan Nacional de $\mathrm{I}+\mathrm{D}+\mathrm{i})$.

RESUMEN: El turismo se ha convertido en un elemento clave en la planificación urbana, desarrollo y evoluciones socioculturales de muchas ciudades. El objetivo de esta investigación es analizar el estado de la cuestión de la metamorfosis acaecida en el turismo y las transformaciones urbanas de las ciudades turísticas en el contexto de la COVID-19, así como las propuestas para una reactivación en forma de turismos de proximidad más alineados con los ODS de Naciones Unidas. El método utilizado está basado en la aplicación del Proceso de Desarrollo del Conocimiento - Constructivista (ProKnow-C), proporcionando un procedimiento estructurado, riguroso y que minimiza el uso de la aleatoriedad y la subjetividad en el proceso de revisión bibliográfica. 
Como resultados, se obtienen 30 artículos relevantes y alineados con el tema de investigación, posibilitando el identificar los principales enfoques propuestos por los autores de este portfolio bibliográfico en relación a la problemática planteada. Esto permite constar la importante proliferación de publicaciones científicas que abordan esta temática, en un corto periodo de tiempo, y la ausencia de un modelo consensuado que permita medir tales fenómenos de forma integral, viéndose además como una oportunidad para repensar el turismo de proximidad como foco de un modelo turístico sostenible.

Palabras clave: Turismo sostenible, overtourism, COVID-19, proximidad, turismo urbano.

RESUM: El turisme s'ha convertit en un element clau en la planificació urbana, el desenvolupament i les evolucions socioculturals de moltes ciutats. L'objectiu d'aquesta investigació és analitzar l'estat de la qüestió de la metamorfosi esdevinguda en el turisme i les transformacions urbanes de les ciutats turístiques en el context de la COVID-19, així com les propostes per a una reactivació en forma de turismes de proximitat més alineats amb els ODS de Nacions Unides. El mètode utilitzat està basat en l'aplicació del Procés de Desenvolupament del Coneixement - Constructivista (ProKnow-C), proporcionant un procediment estructurat, rigorós i que minimitza l'ús de l'aleatorietat i la subjectivitat en el procés de revisió bibliogràfica. Com a resultats, s'obtenen 30 articles rellevants i alineats amb el tema d'investigació, possibilitant l'identificar els principals enfocaments proposats pels autors d'aquest portafoli bibliogràfic en relació a la problemàtica plantejada. Això permet constatar la important proliferació de publicacions científiques que aborden aquesta temàtica, en un curt període de temps, i l'absència d'un model consensuat que permeta mesurar tals fenòmens de manera integral, veient-se a més com una oportunitat per a repensar el turisme de proximitat com a focus d'un model turístic sostenible.

Paraules clau: Turisme sostenible, overtourism, COVID-19, proximitat, turisme urbà. 
ABSTRACT: Tourism has become a key element in the urban planning, development and socio-cultural evolution of many cities. The objective of this research is to analyze the state of the art of the metamorphosis that has occurred in tourism and the urban transformations of tourist cities in the context of COVID19 , together with proposals for reactivation in the form of local tourism in line with the United Nations SDGs. The study applies the Knowledge Development Process - Constructivist (ProKnow-C) methodology, which provides a structured, rigorous procedure that minimizes randomness and subjectivity in the literature review process. Thirty relevant articles aligned with the research topic were obtained for the literature review, from which the main approaches proposed by the authors were identified in relation to the research problem. This result reflects the significant proliferation of scientific publications addressing this issue, in a short period of time, and the absence of a consensual model that allows such phenomena to be measured holistically; it also provides an opportunity to rethink local tourism as a focus for a sustainable tourism model.

KeYwORDs: Sustainable tourism, overtourism, COVID-19, proximity, urban tourism.

\section{Introducción y estado de la cuestión}

as grandes ciudades son destinos turísticos caracterizados por poseer
un gran dinamismo y, además, el turismo ha adquirido un gran peso en estos espacios urbanos (Vargas et al. 2015). Este turismo está en constante evolución, adaptándose a las actuales tendencias que el consumidor turístico demanda. Sin embargo, los últimos veinte años han supuesto un desafío en la gestión del turismo sostenible para los residentes, turistas y visitantes, ya que desde hace menos de una década la población local ha cambiado de forma radical sus percepciones, convirtiendo el turismo de masas en un problema político local que llega incluso a afectar a su población (Goodwin 2017).

Como consecuencia de esta concentración de la actividad turística, los espacios han estado sometidos a complejas transformaciones entre las que se incluyen el sobreturismo (overtourism) o la gentrificación, que implican una transformación funcional de los mismos (De la Calle Vaquero 2019). Este 
crecimiento está siendo foco de análisis, ya que las partes interesadas, aun teniendo algunos roles similares, tienen visiones del mundo muy diferentes (Boom et al. 2021). Además, la demanda descontrolada junto con una concentración del turismo en destinos particulares impacta negativamente tanto en los territorios como en las comunidades locales (Capocchi et al. 2019).

Este impacto que el sobreturismo (overtourism) tiene, de forma real o percibida, en los destinos, acarrea importantes consecuencias ecológicas, sociales y culturales, conduciendo a formas de turismo social y ecológicamente insostenibles, por lo que se vuelve más urgente la necesidad de que el sector se actualice o se diversifique hacia un modelo que equilibre los intereses económicos con los sociales y ecológicos (Benner 2020).

La sostenibilidad es uno de los principales retos de la sociedad, ya que los brotes más dañinos corresponden a grandes industrias con intereses internacionales, lo que hace más compleja la gestión ambiental y la eliminación del enfoque directo (Cabello et al. 2021). El éxito a largo plazo del turismo urbano sostenible requiere preservar la autenticidad de la ciudad, y hacerlo requiere intervenciones políticas, basadas en una visión a largo plazo, pronósticos y participación de las partes interesadas (Boom et al. 2021b). Este desarrollo responde a las necesidades tanto de turistas como de regiones receptoras, además de estar enfocado hacia la gestión de los recursos, por lo que va dirigido a satisfacer la actividad de ocio de los visitantes, a la vez que cuide las necesidades de la comunidad receptora gracias a los beneficios generados (Linares y Garrido 2014).

Sin embargo, la pandemia causada por la COVID-19 ha hecho que, en el contexto mundial, se presente la urgente necesidad de configurar ciudades inclusivas y resilientes (Cabral y Diez 2020). Además, el turismo en España ha jugado un papel muy importante en las principales crisis ocurridas, al ser uno de los principales sectores que ha contribuido a la recuperación económica tras cada una de ellas (Benito 2016). Las investigaciones anteriores sobre crisis y desastres muestran patrones similares, y las teorías existentes a menudo pueden explicar los fenómenos que se observan a día de hoy (Zeker y Kock 2020). La cuestión que surge actualmente en la industria, se centra en si deberíamos volver al statu quo anterior a la pandemia lo antes posible y haciendo uso de la financiación pública, o si deberíamos aprovechar los 
efectos de esta como una oportunidad para modificar, parcial o totalmente, las condiciones económicas y sociales que llevaron al sobreturismo (Montanari 2020).

Este periodo se contempla, por tanto, como una oportunidad para reconsiderar una transformación del sistema turístico global, enfocándolo hacia uno más alineado con los Objetivos de Desarrollo Sostenible para 2030 (ODS) de las Naciones Unidas (Rivera García y Pastor Ruíz 2020). Como indican Pons et al. (2020), la sostenibilidad turística no implica únicamente aportar una solución equilibrada a la rentabilidad económica, sino también al bienestar social y al equilibrio ecológico, es decir, soluciones prácticas para un turismo más sostenible.

Un ejemplo de estas propuestas puede ser prolongar la duración de la estancia, abastecerse en mercados más cercanos, repensar la comida para optar por productos orgánicos (y de proximidad) y de temporada, avanzar hacia un modelo de alto valor añadido, dar beneficio local y no a plataformas globales de propiedad extranjera, abandonar los viajes intensivos en carbono, por ejemplo, en cruceros, lograr reducir la estacionalidad, pero sobre todo, viajar de manera que sea económicamente viable, preservando el tejido social de las ciudades (Gössling y Higham 2020). Surge además el turismo de proximidad como una de estas propuestas para reconducir el sector (Romagosa, 2020). Este turismo no sólo está asociado a una práctica de turismo más sostenible (Díaz, 2017), sino que además se trata de una de las consecuencias más probables de la crisis actual (Navarro, Ortega y Torres 2020). Esto se debe en gran medida a la existencia de una mayor conciencia social y ambiental por parte de los turistas, que en muchas ocasiones optarán por viajar a destinos más cercanos a su lugar de residencia, lo que sumado a las posibles restricciones en los viajes internacionales (al menos de forma temporal) ayudará a la promoción de este turismo a la vez que se promociona un turismo más sostenible (Romagosa 2020b).

El turismo es considerado clave en la planificación urbana, desarrollo y transformaciones socioculturales de muchas ciudades, pero ¿es el sobreturismo una consecuencia de las transformaciones urbanas? ¿Podemos considerar el turismo de proximidad como una estrategia para lograr un destino más accesible y sostenible durante y tras el COVID-19? ¿Cuándo se con- 
sidera que estamos en un nivel óptimo de sostenibilidad y cómo lo medimos? ¿Se trata únicamente de indicadores medioambientales o hay algo más?

En aras de dar respuesta a estas cuestiones, nuestra investigación tiene como objetivo analizar el estado del arte de la metamorfosis que ha experimentado el turismo y las transformaciones urbanas de las ciudades turísticas en el contexto del COVID-19. Esta investigación presenta la aplicación detallada del Proceso de Desarrollo del Conocimiento - Metodología constructivista (Proknow-C), mediante la creación de un portafolio bibliográfico relevante a través del análisis bibliométrico, logrando seleccionar aquellos trabajos altamente alineados con este tema investigado. Esto constituye un estudio analítico del conocimiento acumulado que parte de la investigación documental, gracias a la que podremos llevar a cabo el estudio de un conocimiento acumulado dentro de un área específica, dividiéndose, normalmente, en: contextualización, clasificación y categorización (Molina 2005). Esta modalidad tiene como objetivo inventariar y sistematizar la producción en un área del conocimiento, ejercicio que no debe quedarse tan solo en el inventario, sino trascender más allá, pues permite hacer una reflexión profunda sobre las tendencias y vacíos en un área específica (Vargas y Calvo 1987).

Los hallazgos de este estudio permitirán constar la importante proliferación de publicaciones científicas que abordan esta temática, en un corto periodo de tiempo, además de la ausencia de un modelo consensuado que permita medir tales fenómenos de forma integral. Esto se ve, además, como una oportunidad para repensar el turismo de proximidad como foco de un modelo turístico sostenible, ya que el desarrollo masivo y descontrolado del turismo tiene repercusiones negativas para el medio natural. El turismo sostenible implica no solo dar una solución equilibrada a la rentabilidad económica, sino también al bienestar social y al equilibrio ecológico, enfocándolo hacia uno más alineado con los ODS de Naciones Unidas (Rivera y Pastor 2020). La revisión exhaustiva de la literatura efectuada nos desvela el conocimiento acumulado sobre estos aspectos y hace explícito sus antecedentes, causas, modelos de detección y soluciones que permitan avanzar hacia un desarrollo turístico más sostenible. Además, nos va a permitir conocer la simbiosis antes mencionada, así como las propuestas de reactivación en forma de turismo de proximidad durante y tras la COVID-19. 


\section{Metodología}

Una búsqueda o revisión de literatura consiste en un método sistémico, explícito y reproducible que permite identificar, evaluar y sintetizar el trabajo ya existente y producido por otros investigadores (Fink 2019). De acuerdo con Fink (2019), Boland et al. (2017), Hart (2017) Gough et al. (2017) y Heyvaert et al. (2016), hay seis fases en la creación de una revisión de literatura, cuyo objetivo es determinar el estado actual de búsqueda o discusión científica en cuanto a un campo particular de conocimiento (Kuckartz \& Rädiker 2019):

1. Formular preguntas y objetivos de investigación, es decir, determinar qué se quiere conseguir.

2. Seleccionar las bases de datos bibliográficas, que hoy en día son, en su mayoría, online.

3. Determinar los términos de búsqueda.

4. Aplicar criterio práctico y metodológico para la selección de literatura científica de alta calidad.

5. Llevar a cabo la revisión de los textos completos, definiendo las variables, codificando, añadiendo notas y resúmenes.

6. Sintetizar los resultados y conclusiones.

Para la selección del marco teórico y construcción de los conocimientos necesarios (puntos 2, 3 y 4) el método utilizado se basó en la metodología Proknow-C (Knowledge Development Process - Constructivist), propuesta por Ensslin et al. (2010), que consiste en estructurar una revisión de la literatura para construir conocimiento y seleccionar revistas para su fundamentación teórica (Vieira et al. 2019).

El objetivo fue realizar una revisión sistémica con el fin de localizar los estudios más relevantes a partir de las palabras clave de esta investigación (Ravindran y Shankar 2015), así como un análisis bibliométrico de la producción científica al respecto (Duque y Cervantes-Cervantes 2019). El estudio se diseñó para llenar los vacíos de revisiones anteriores, examinando el estado, las tendencias y las posibles áreas de investigación futura, de forma que ofrezca una visión general sobre el sobreturismo (overtourism) como consecuencia de las transformaciones urbanas en las grandes ciudades, y los 
procesos de reactivación en forma de turismos de proximidad para lograr un destino más sostenible y accesible a través de modelos de gestión turística que aportan focos innovadores (Jurado, Quintana y Rodriguez 2020). Por ello, esta investigación se realizó a través de un diseño cualitativo e interpretativo, que determinó el procedimiento de selección, acceso y registro de la muestra documental (Vargas, Higuita y Muñoz 2015). De esta forma se presenta un panorama general de todos los ejes de búsqueda investigados, con un enfoque interdisciplinario y comprensivo (Jurado, Quintana y Rodriguez 2020b). El diseño del proceso metodológico se organiza en tres fases, que se explican a continuación.

En las dos primeras fases los pasos seguidos, basándonos en los propuestos por de Carvalho et al. (2020), fueron los siguientes:

1. Definición de palabras clave.

2. Definición de bases de datos.

3. Búsqueda de artículos en las bases de datos con las palabras clave.

4. Aplicación de prueba de adherencia de palabras clave.

5. Eliminación de artículos repetidos.

6. Alineación por lectura del título.

7. Alineación como reconocimiento científico, por ejemplo, número de citas y artículos recientes.

8. Alineación leyendo el resumen.

9. Alineación por lectura completa de artículos: análisis bibliométricos y sistemáticos descriptivos.

En la primera fase, se establecen los ejes de investigación o búsqueda. Debido a que uno de los objetivos principales es analizar la simbiosis entre los diferentes términos para analizar una posible transformación del sistema turístico global, enfocándolo hacia uno más alineado con los ODS de Naciones Unidas, se establecieron cuatro ejes de investigación o búsqueda: "sobreturismo y turismo sostenible", "COVID-19 y turismo sostenible", "turismo de proximidad y turismo sostenible" y "turismo urbano y turismo sostenible". 
Para estos ejes de investigación, se establecen las correspondientes palabras clave: para el eje "sobreturismo y turismo sostenible", overtourism en inglés, y sobreturismo, en español, para más tarde en el proceso de filtrado seleccionar aquellos que relacionan dicho término con turismo sostenible. Para "COVID-19 y turismo sostenible", debido a que en este caso se trata de un término demasiado amplio, a fin de realizar dicha conexión con turismo sostenible se opta por introducir ambos términos combinados en el buscador al objeto de acotar el número de trabajos a revisar, por lo que se estable como palabras clave COVID-19 y sustainable tourism en inglés, y COVID-19 y turismo sostenible en español. Para "turismo de proximidad y turismo sostenible" se establece los términos proximity en inglés y proximidad en español. Por último, para el eje "turismo urbano y turismo sostenible" al igual que ocurría con el término sobreturismo (overtourism), se trata de un término demasiado amplio, por lo que se optó como palabras clave turismo urbano y turismo sostenible en español, y urban tourism y sustainable tourism en inglés.

El carácter multidisciplinar que presenta el turismo llevó a seleccionar las bases de datos Web of Science, Scopus y Google Académico, por ser buscadores especializados en artículos de revistas científicas, enfocados en el mundo académico, y que almacenan un amplio conjunto de trabajos de investigación científica de distintas disciplinas y en distintos formatos de publicación. Se utilizaron estas bases ya que, a pesar de que Google Académico causa en ocasiones ciertos errores y limitaciones consecuencia de su enfoque automatizado para la indexación de documentos (Delgado, Robinson y Torres 2014), ha demostrado ser una fuente confiable, además de tener una buena cobertura en diferentes disciplinas, en especial en Humanidades y Ciencias Sociales (Delgado, Orduna y Martin 2018).

Estos instrumentos jerarquizan los resultados, aunque también usan como señal de calidad la revista en la que se ha publicado, permitiendo el acceso a copias físicas o digitales de artículos, ya sea online o en bibliotecas. Además, las arañas de Google académico rastrean constantemente los sitios web de universidades, editoriales científicas, repositorios, catálogos y cualquier otro espacio web donde encontrar materiales de tipo académico, independientemente de su tema o idioma. A diferencia del acumulativo y naturaleza 
selectiva de Web of Science y Scopus, Google Académico es dinámico, ya que refleja el estado de la web, que es visible para sus robots de búsqueda y para la mayoría de los usuarios en un momento específico en el tiempo (Delgado, Orduna y Martin 2018b).

Se utilizaron los campos de búsqueda: título, resumen y palabras clave, y se restringieron los años a los periodos 1900-2021, para artículos de revistas científicas. Completando esta primera fase y para obtener el banco de publicaciones bruto, se realizó una prueba de adherencia de artículos con palabras clave, que consistió en seleccionar al azar varios entre el citado banco obtenido, a fin de identificar las palabras clave de cada uno de ellos, para determinar si en el título, las palabras clave o resumen se encuentran las definidas en esta investigación, de modo que el portafolio esté lo más alineado posible (dos Santos Matos \& Petri 2015). Además, se llevó a cabo una primera criba en cada eje de investigación, eliminando aquellos artículos duplicados en las tres bases de datos utilizadas, y se procedió a realizar una exclusión por lectura de título dentro de cada uno de estos ejes.

La segunda fase comienza con la exclusión de aquellos que estuviesen duplicados una vez obtenido el primer banco de artículos brutos de todos los ejes de investigación. A continuación, se procedió a la lectura de los títulos, y a partir de ahí se verificó el reconocimiento científico mediante una búsqueda de la cantidad de citas que tenía cada artículo en las bases de datos consultadas. Aquellos descartados por su falta de reconocimiento científico, fueron sometidos a una reevaluación que se detalla a continuación (figura 1): 


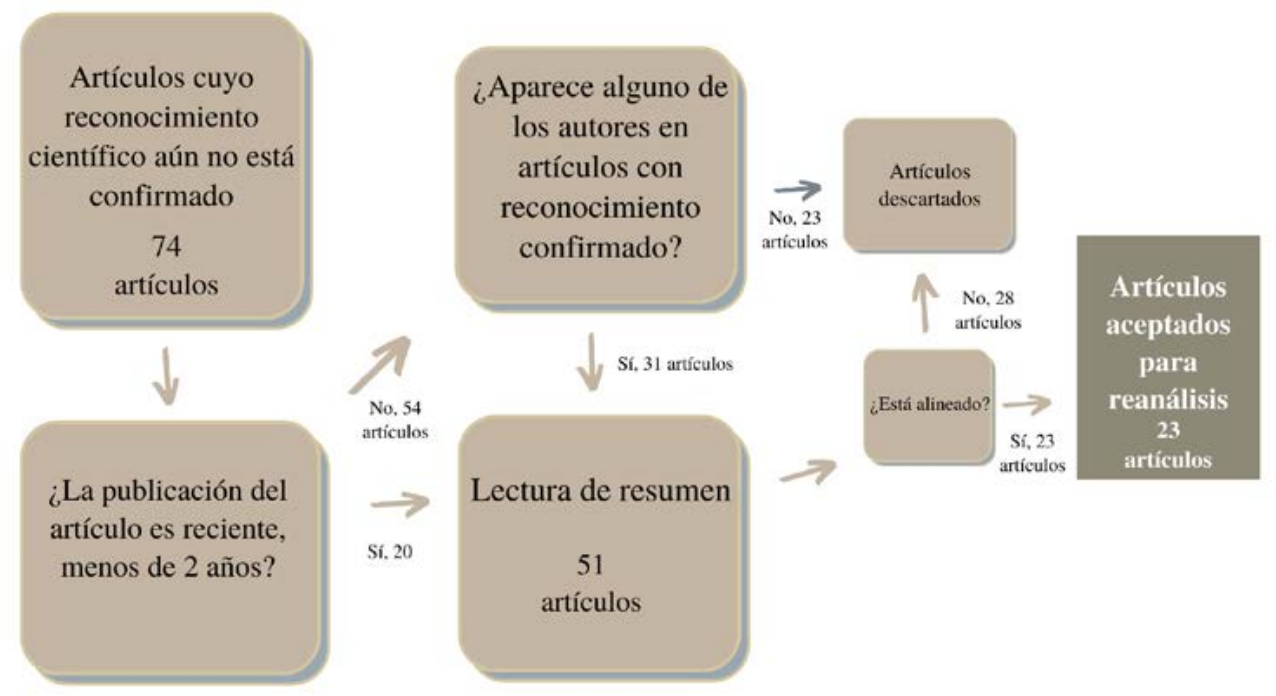

Figura 1: Filtro para seleccionar artículos con reconocimiento científico menor adaptado de Esslin et al. (2010).

Posteriormente, se procedió a la lectura de los resúmenes de los artículos, para seleccionar los alineados con el tema de investigación. Por último, se realizó la lectura completa de los textos, y se llevaron a cabo los análisis bibliométricos y sistemáticos descriptivos (Vieira et al. 2019, Ensslin et al. 2010, Ravindran y Shankar 2015).

Para realizar la revisión sistemática de esta literatura se partió de una pregunta explícita y claramente definida, buscando una interpretación del análisis y reporte de los resultados apropiados (Ravindran y Shankar 2015). En este caso, se trata de desvelar el conocimiento acumulado sobre los términos overtourism, COVID-19, proximidad y turismo urbano, y hacer explícito sus antecedentes, causas, modelos de detección y soluciones que permitan avanzar hacia un desarrollo turístico más sostenible. Una vez efectuados estos pasos, se dispone de los 30 artículos alineados con el objetivo de investigación para proceder a su análisis.

A fin de continuar con los puntos 5 y 6 propuestos por Kuckartz y Rädiker (2019), y coincidiendo con la tercera fase de nuestra investigación, se procedió a agrupar las ideas más relevantes en una tabla de autores a partir de los informes y comentarios de texto recogidos durante la lectura de los 
documentos. Se resaltaron aquellos aspectos que más se repetían, además de establecer interrelaciones entre los autores, para tratar de reforzar, a través de las aportaciones de este trabajo de investigación, proposiciones, acciones e innovaciones que permitieran clarificar posibles procesos que contribuyeran a la identificación de estos términos. Para lograrlo se utilizó el programa MAXQDA, que permitió extraer tanto las palabras que más se repetían a lo largo del cuadro-resumen de los autores más representativos que han abordado esta problemática, como establecer interrelaciones entre las diferentes palabras clave de nuestra investigación y las aportaciones de los autores.

En primer lugar, se analizó la frecuencia de términos en el documento para proceder a elaborar una nube de palabras, eliminando para ello aquellas que no se consideraron relevantes para la investigación o poseyesen una frecuencia inferior a 6 por no considerarse destacable para el estudio. Seguidamente se comprobó la frecuencia con la que aparecía cada una de las palabras clave en la tabla de autores (Rädiker y Kuckartz 2021). A continuación, se establecieron los códigos y, una vez hecho, se categorizaron en la tabla de autores (Cazau 2004) para analizar la interrelación entre ellos. Posteriormente, se observó la frecuencia de aparición de cada código a lo largo del documento, así como la distribución de cada uno (Sgier 2012). Por último, se realizó el análisis de resultados y las conclusiones, dando respuesta a los interrogantes de investigación sobre la metamorfosis del turismo y las transformaciones urbanas en las grandes ciudades en el contexto de la COVID-19, las propuestas para una reactivación en forma de turismos de proximidad, así como aquellos modelos y soluciones que permitan avanzar hacia un desarrollo turístico más sostenible. Además, permitió retocar la tabla de autores de forma que quedaran reflejadas clara y visualmente en la misma las palabras clave y los resultados de la codificación de cada autor.

\section{Análisis y resultados}

Los resultados más relevantes para las palabras clave buscadas se listan, en primer lugar, según el ranking del autor, el número de referencias que lo enlazan y su relevancia respecto de otra literatura académica, así como el ranking de la propia publicación en que aparece el artículo. Por ello, en una búsqueda preliminar, se comenzó introduciendo: overtourism, COVID-19, 
proximidad y turismo urbano, siempre en combinación cada una de ellas con otra palabra clave: turismo sostenible.

Se comienza con el primer eje de investigación, sobreturismo (overtourism) y turismo sostenible. En primer lugar, se realizó una búsqueda del término overtourism, de la que se obtuvieron 9340 resultados en Google Académico, 259 en Web of Science y 256 en Scopus. A continuación, se filtró tanto en Web of Science como en Scopus para obtener únicamente los artículos científicos, obteniéndose 227 resultados en Web of Science y 203 en Scopus (consultado en 5 de marzo de 2021). Tras realizar la prueba de adherencia y la eliminación por artículos repetidos en todas las bases de datos, se descartaron un total de 4250. Posteriormente, se procedió a la alineación por lectura de título de los 5520 restantes, de los que se descartaron 5470 por no estar alineados con el objetivo de la investigación, resultando finalmente en 50 trabajos, todos ellos combinando el término overtourism con turismo sostenible.

Se continúa con el eje de investigación COVID-19 y turismo sostenible. Con las palabras clave establecidas (COVID-19 y sustainable tourism) se obtuvo 34400 resultados en Google Académico, 95 en Web of Science y 121 en Scopus, mientras que en lengua castellana (COVID-19 y turismo sostenible) las obras a analizar eran 6420 en Google Académico, ninguno en Web of Science y 2 en Scopus (aunque uno de ellos estaba en inglés). A continuación, se filtró tanto en Web of Science como en Scopus para obtener únicamente los artículos científicos, obteniéndose 89 resultados en Web of Science y 93 en Scopus (consultado en 5 de marzo de 2021). Tras realizar la prueba de adherencia y la eliminación por artículos repetidos en todas las bases de datos, se descartaron un total de 35230. Posteriormente, se procedió a la alineación por lectura de título de los 5772 restantes, de los que se descartaron 5722 por no estar alineados con el objetivo de la investigación, resultando finalmente en 50 trabajos.

A continuación, se realizó el mismo procedimiento con el eje turismo de proximidad y turismo sostenible. Los términos proximidad y proximity al introducir en español en Google Académico alcanzan 65700 resultados, ningún resultado en Web of Science y 4 resultados en Scopus (se introdujeron los términos en español, pero los 4 resultados eran en inglés); introduciendo 
el término en inglés se obtuvo 300000 artículos en Google Académico, 626 en Web of Science y 754 en Scopus. A continuación, se filtró tanto en Web of Science como en Scopus para obtener únicamente los artículos científicos, obteniéndose 561resultados en Web of Science y 597 en Scopus (consultado en 6 de marzo de 2021). Sin embargo, en Google Académico para realizar la conexión con turismo sostenible se opta por introducir este término en el buscador a fin de acotar el número de trabajos a revisar. Con las nuevas palabras clave (proximity y sustainable tourism) se obtiene 77000 registros, mientras que en lengua castellana (proximidad y turismo sostenible) las obras a analizar eran 22400 (consultado en 7 de marzo de 2021). Tras realizar la prueba de adherencia y la eliminación por artículos repetidos en todas las bases de datos, se descartaron un total de 77456. Posteriormente, se procedió a la alineación por lectura de título de los 23102 restantes, de los que se descartaron 23052 por no estar alineados con el objetivo de la investigación, resultando finalmente en 50, y en los que aparece un nexo al término turismo sostenible.

Por último, se realizó el mismo procedimiento con el término turismo urbano y urban tourism, obteniendo al introducir el término en español 574000 resultados en Google Académico, ningún resultado en Web of Science y 30 resultados en Scopus (se introdujeron los términos en español, pero los resultados eran casi en su totalidad en inglés); introduciendo el término en inglés se obtuvo 2980000 artículos en Google Académico, 6359 en Web of Science y 7767 en Scopus. Al igual que en el caso de sobreturismo, debido a que en este caso se trata de un término demasiado amplio, para realizar dicha conexión con turismo sostenible se opta por introducir este último en el buscador en todas las bases de datos, a fin de acotar el número de trabajos a revisar. Con las nuevas palabras clave (turismo urbano y turismo sostenible) se obtuvo 101000 resultados en Google Académico, ninguno en Web of Science y ninguno en Scopus, mientras que en inglés (urban tourism y sustainable tourism) las obras a analizar eran 1440000 en Google Académico, 1139 en Web of Science y 1403 en Scopus. A continuación, se filtró tanto en Web of Science como en Scopus para seleccionar únicamente los artículos científicos, obteniéndose 827 resultados en Web of Science y 910 en Scopus (consultado en 6 de marzo de 2021). Debido al elevado número de artículos encontrados en Google Académico (1541000 entre inglés y español), se procedió a acotar en esta búsqueda el rango de años a los últimos 7 años, por 
considerarse los más relevantes para la investigación (2014-2021). Los resultados fueron de 206000 en inglés, y 15400 en español. Tras realizar la prueba de adherencia y la eliminación por artículos repetidos en todas las bases de datos, se descartó un total de 211001. Posteriormente, se procedió a la alineación por lectura de título de los 12136 restantes, de los que se descartaron 12086 por no estar alineados con el objetivo de la investigación, resultando finalmente en 50 trabajos.

Esta primera criba dio como resultado 200 artículos (50 de cada palabra clave relacionada con turismo sostenible), de los que se realizó un proceso de filtrado del banco de artículos en bruto, comenzando con la exclusión de aquellos que aparecían duplicados, descartando 7. En la segunda etapa se volvió a alinear por lectura del título los 193 restantes, resultando preseleccionados 150 trabajos.

Se procedió a una tercera alineación, esta vez por reconocimiento científico, de la que se descartaron 74, pasando los 76 restantes a la siguiente fase. Sin embargo, esos 74 descartados pasaron por una reevaluación (figura 1), en la que se reincorporaron 23 artículos para continuar con su análisis, que se añaden a los 76 previamente mencionados, sumando un total de 99 artículos.

Posteriormente, se leyeron los resúmenes de los 99 artículos, para hacer la selección de aquellos que estaban alineados con el tema de investigación, descartando 19. Por último, se accedió a los textos de los 80 restantes para su lectura y revisión de la información requerida y se llevaron a cabo los análisis bibliométricos y sistemáticos descriptivos, resultando en los 30 artículos finales de todo el proceso (Vieira et al. 2019, Ensslin et al. 2010, Ravindran y Shankar 2015).

El plan de análisis consistió en dos lecturas: una lineal que exigió la revisión consecutiva de la información obtenida en las fuentes bibliográficas, y otra transversal que permitió la comparación de las fuentes a partir de las categorías aplicadas para identificar las repeticiones, vacíos, confirmaciones, ampliaciones, falencias, así como la calidad y cualidad de la información sobre el objeto de investigación. Las observaciones sobre los hallazgos en las fuentes se reseñaron en memos de investigación y comentarios de texto que se usaron posteriormente para la elaboración de una tabla, resultado de la aportación de los autores en los 30 artículos de investigación de la búsqueda. 
Se pasó a introducir el documento con la tabla de aportaciones de los autores en MAXQDA. En primer lugar, se analizó la frecuencia de palabras en el documento para elaborar una nube (figura 2). Por no considerarse destacable para el estudio se eliminaron aquellas no relevantes para la investigación y las que poseen una frecuencia inferior a 6 (Rädiker y Kuckartz 2021).

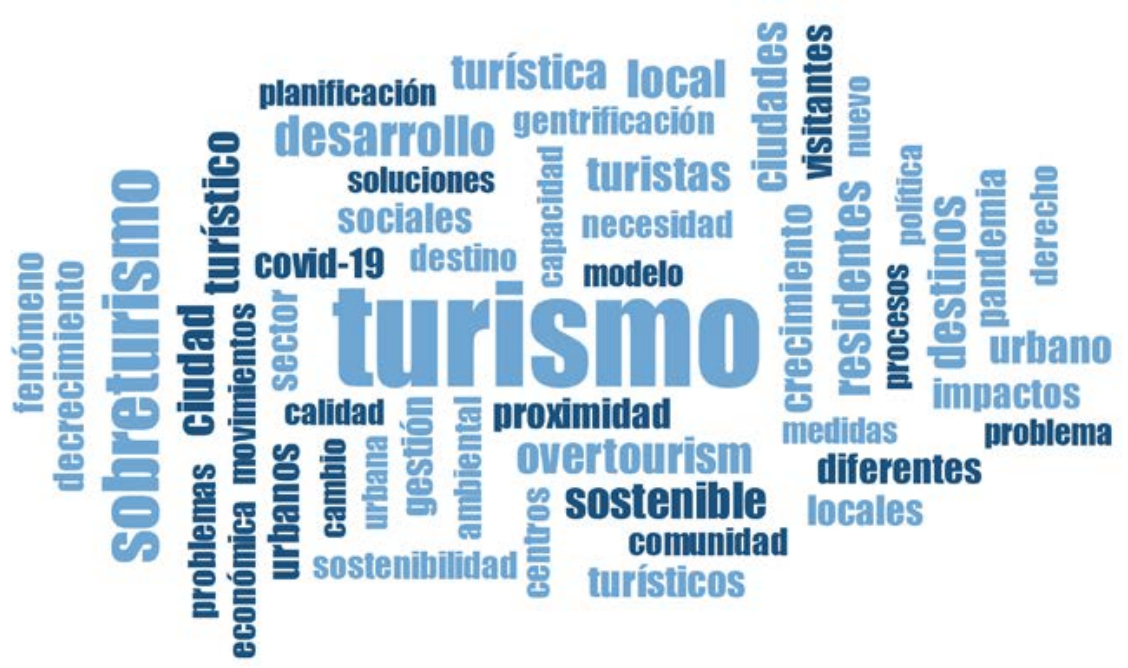

Figura 2: Frecuencia de palabras relevantes en la tabla de autores. Fuente: Autores desde MAXQDA.

A continuación, se comprobó la frecuencia con la que aparecía cada una de las palabras clave en la tabla de autores. Cabe puntualizar que se ha considerado como palabra clave tanto el término overtourism como su traducción al español, sobreturismo, ya que dependiendo del autor se ha hecho referencia a una u otra, aunque siempre hablando del mismo fenómeno (figura 3):

\begin{tabular}{|lllll}
\hline Overtourism / Sobreturismo & COVID-19 & Turismo sostenible & Proximidad & Turismo urbano \\
\hline 66 & 11 & 11 & 11 & 15
\end{tabular}

Figura 3: Frecuencia de nuestras palabras clave en la tabla de autores. Fuente: Autores desde MAXQDA. 
Ya que a lo largo de la revisión de la literatura dos de los aspectos a destacar son la problemática existente y las aportaciones que realiza cada autor para abordarla, se han añadido una $6^{\mathrm{a}}$ y $7^{\mathrm{a}}$ categoría denominadas "problemática" y "aportaciones" para interrelacionar estas variables con el resto de categorías. Una vez establecidos los códigos, se procedió a la categorización de la tabla de autores (Cazau 2004) para analizar la interrelación entre ellas, en especial, asegurarnos que la variable "turismo sostenible" aparece reflejada a lo largo de toda la tabla, ya que el objetivo es desvelar el conocimiento acumulado sobre estos conceptos y analizar modelos de detección y soluciones que permitan avanzar hacia un desarrollo turístico más sostenible. A continuación, se muestra la interrelación entre los códigos, mostrando como todos ellos van unidos al código Turismo Sostenible (figura 4):

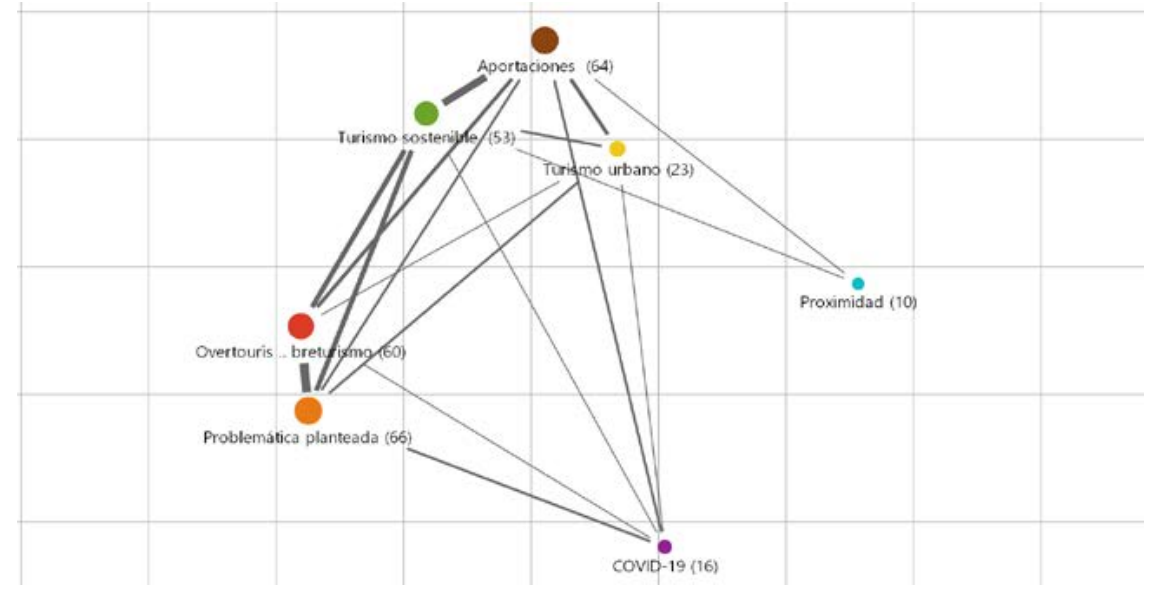

Figura 4: Interrelación entre los códigos. Fuente: Autores desde MAXQDA.

En la siguiente imagen (figura 5) se observa la frecuencia de aparición de cada código a lo largo del documento. Como datos destacables cabe señalar la poca proliferación de artículos sobre COVID-19 y turismo de proximidad en relación con Turismo Sostenible. También destaca que los códigos de overtourism (rojo) y turismo sostenible (verde) son los que, dejando a un lado los de problemática (naranja) y aportaciones (marrón), más aparecen codificados a lo largo de la tabla de autores, tal y como se observa en la categorización: 


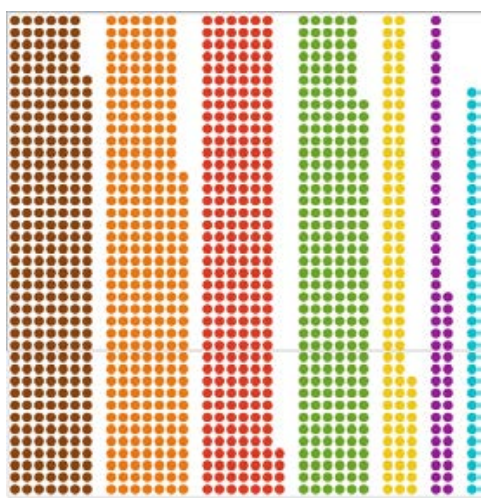

Figura 5: Interrelación entre los códigos. Fuente: Autores desde MAXQDA.

Finalmente, se procedió a la elaboración de una segunda tabla en la que reflejar las palabras clave de cada documento de acuerdo a los resultados de la codificación (tabla 1).

Tabla 1: Tabla de autores y palabras clave.

\begin{tabular}{|l|l|}
\hline AUTOR/ES & PALABRAS CLAVE \\
\hline $\begin{array}{l}\text { Alexandrova, Aigina, Kaverzin y Sheresheva } \\
(2019)\end{array}$ & $\begin{array}{l}\text { Turismo urbano, sobreturismo } \\
\text { (overtourism), turismo sostenible }\end{array}$ \\
\hline Amore, Falk y Adie (2020) & $\begin{array}{l}\text { Sobreturismo (overtourism), turismo } \\
\text { sostenible, turismo urbano }\end{array}$ \\
\hline Benner (2020) & $\begin{array}{l}\text { Sobreturismo (overtourism), turismo } \\
\text { sostenible }\end{array}$ \\
\hline Bertocchi y Visentin (2019) & $\begin{array}{l}\text { Sobreturismo (overtourism), turismo } \\
\text { urbano, turismo sostenible }\end{array}$ \\
\hline $\begin{array}{l}\text { Bertocchi, Camatti, Giove y van der Borg } \\
(2020)\end{array}$ & $\begin{array}{l}\text { Sobreturismo (overtourism), turismo } \\
\text { sostenible }\end{array}$ \\
\hline $\begin{array}{l}\text { Boom,Weijschede, Melissen, Koens y Mayer } \\
(202 \text { I) }\end{array}$ & $\begin{array}{l}\text { Turismo urbano, sobreturismo } \\
\text { (overtourism), turismo sostenible }\end{array}$ \\
\hline $\begin{array}{l}\text { Cabello, Navarro, Thiel, Rodríguez y Ruiz } \\
(202 I)\end{array}$ & Turismo sostenible, turismo urbano \\
\hline Cabral y Diez (2020) & $\begin{array}{l}\text { COVID- I9, turismo urbano, turismo } \\
\text { sostenible, proximidad }\end{array}$ \\
\hline Capocchi,Vallone, Pierotti y Amaduzzi (20I9) & $\begin{array}{l}\text { Sobreturismo (overtourism), turismo } \\
\text { sostenible }\end{array}$ \\
\hline Capocchi,Vallone, Amaduzzi y Pierotti (2020) & $\begin{array}{l}\text { Sobreturismo (overtourism), turismo } \\
\text { sostenible }\end{array}$ \\
\hline Cheung y Li, (20I9) & $\begin{array}{l}\text { Sobreturismo (overtourism), turismo } \\
\text { sostenible }\end{array}$ \\
\hline Crossley (2020) & $\begin{array}{l}\text { COVID- I9, sobreturismo (overtourism), } \\
\text { turismo sostenible y turismo urbano }\end{array}$ \\
\hline
\end{tabular}




\begin{tabular}{|c|c|}
\hline De la Calle, M. (2019) & $\begin{array}{l}\text { Sobreturismo (overtourism), turismo } \\
\text { urbano, COVID-19, turismo sostenible }\end{array}$ \\
\hline De la Calle, García y Mendoza (202I) & $\begin{array}{l}\text { Turismo urbano, COVID-19, sobreturismo } \\
\text { (overtourism), turismo sostenible }\end{array}$ \\
\hline Díaz y Llurdés (2013) & Proximidad, turismo sostenible \\
\hline Díaz y Jover (202I) & $\begin{array}{l}\text { Turismo urbano, sobreturismo } \\
\text { (overtourism), turismo sostenible }\end{array}$ \\
\hline $\begin{array}{l}\text { Fedyk, Sołtysik, Olearnik, Barwicka, y Mucha } \\
(2020)\end{array}$ & $\begin{array}{l}\text { Turismo sostenible, sobreturismo } \\
\text { (overtourism), turismo urbano }\end{array}$ \\
\hline Fletcher, Murray, Blanco y Blázquez (2019) & $\begin{array}{l}\text { Sobreturismo (overtourism), turismo } \\
\text { sostenible }\end{array}$ \\
\hline García, Ivars y Mendoza (2019) & $\begin{array}{l}\text { Turismo urbano, turismo sostenible, } \\
\text { sobreturismo (overtourism) }\end{array}$ \\
\hline García y Ruíz (2020) & COVID-19, turismo sostenible \\
\hline Jover y Díaz (2020) & $\begin{array}{l}\text { Turismo urbano, sobreturismo } \\
\text { (overtourism), turismo sostenible }\end{array}$ \\
\hline Koh, E. (2020) & $\begin{array}{l}\text { Turismo sostenible, sobreturismo } \\
\text { (overtourism), COVID-19 }\end{array}$ \\
\hline Linares y Garrido (20|4) & Turismo sostenible, turismo urbano \\
\hline Llurdés, Díaz y Romagosa (20|4) & Proximidad, turismo sostenible \\
\hline Milano, Novelli y Cheer (2019) & $\begin{array}{l}\text { Sobreturismo (overtourism), turismo } \\
\text { sostenible, turismo urbano }\end{array}$ \\
\hline Milano, Novelli y Cheer (2019) & $\begin{array}{l}\text { Sobreturismo (overtourism), turismo } \\
\text { sostenible. }\end{array}$ \\
\hline Montanari (2020) & $\begin{array}{l}\text { Sobreturismo (overtourism), COVID-I9, } \\
\text { turismo urbano, turismo sostenible }\end{array}$ \\
\hline Muler, Coromina, y Galí (2018) & $\begin{array}{l}\text { Sobreturismo (overtourism), turismo } \\
\text { sostenible }\end{array}$ \\
\hline Navarro y Capote (2020) & $\begin{array}{l}\text { Turismo sostenible, sobreturismo } \\
\text { (overtourism) }\end{array}$ \\
\hline Romagosa (2020) & $\begin{array}{l}\text { Turismo urbano, turismo sostenible, } \\
\text { proximidad }\end{array}$ \\
\hline
\end{tabular}

Fuente: Elaboración propia.

Uno de los hallazgos a destacar tras el proceso, es que a pesar de que inicialmente los artículos se seleccionaron a través de diferentes ejes de investigación, en una gran parte de ellos aparece otra de las palabras clave que, a pesar de no haber sido utilizada en la estrategia de búsqueda marcada, muestra una correlación entre los temas analizados no detectada a priori.

Por otro lado, también es interesante recalcar la aparición de la palabra clave turismo sostenible codificada en todos los artículos, lo que muestra la consecución de los objetivos de investigación planteados, ya que uno de ellos 
era lograr analizar la relación de los términos sobreturismo (overtourism), turismo urbano, proximidad y COVID-19 con el enfoque hacia un modelo turístico más sostenible.

\section{Discusión y conclusiones}

Este estudio se propuso como objetivo analizar el estado del arte de los cambios que ha experimentado el turismo y las transformaciones urbanas de las ciudades en el contexto de la COVID-19, de forma que nos permitiera conocer la citada simbiosis, así como las propuestas para una reactivación en forma de turismos de proximidad. A través de una exhaustiva revisión de la literatura hemos logrado desvelar el conocimiento acumulado sobre estos conceptos y hacer explícito sus antecedentes, causas, modelos de detección y soluciones que permitan avanzar hacia un desarrollo turístico más sostenible.

A pesar de que existe cada vez más conocimiento acerca de que, en muchos destinos turísticos, la gestión tradicional está ocasionando impactos sociales y ambientales que no son deseables, algunos amenazan, además, con minar la viabilidad económica de la industria turística local. Esto hace que se reduzcan las perspectivas de continuidad de la prosperidad de la misma (Manning et al. 1997). Sin embargo, aún falta por establecer una definición aceptada sobre lo que el turismo sostenible significa y los diferentes puntos de vista sobre el concepto (Berry y Ladkin 1007, Swarbrook 1998), llevando a cuestionar la validez de lograr una sostenibilidad dentro del turismo al estar tan abierta la postura conceptual (Hunter 1997). Es por ello que los destinos turísticos en los que las economías locales son altamente dependientes de la industria turística están especialmente afectados por estos riesgos, y por lo que la OMT ha desarrollado una serie de indicadores de la sostenibilidad aplicable a la industria turística (Manning et al. 1997b).

En las grandes ciudades, el turismo ha sido tradicionalmente un sector importante para la economía urbana, pero tras la citada crisis financiera de 2008 se ha convertido en la principal fuente de ingresos de algunas ciudades (Jover y Diaz 2020). La expansión rápida y desigual del turismo como respuesta a la citada crisis económica se ha producido en paralelo con el aumento del descontento social con respecto al llamado "turismo excesivo" (Fletcher et al. 2019). El desarrollo de este turismo activo en las ciudades ejerce una presión 
creciente tanto en su patrimonio como en su comunidad local, haciendo que los flujos turísticos provoquen hacinamiento y descontento entre los vecinos, y dando lugar al fenómeno del sobreturismo (overtourism) (Alexandrova et al. 2019), en especial cuando pasamos por alto la capacidad de carga de los destinos (Cheung y Li 2019).

El sobreturismo es un término que, en poco tiempo, ha cobrado importancia como uno de los más abordados en relación al turismo, tanto en los medios de comunicación como en la academia, a pesar de no estar aún claramente delineado y permanecer abierto a múltiples interpretaciones (Koens, Postma y Papp 2018). Se trata, además, de un vocablo relativamente nuevo que hace referencia a un fenómeno ya conocido donde la gente local, o los propios turistas, sienten que un lugar es visitado por demasiada gente (Capocchi et al. 2020, De la Calle 2019). Los cambios en el empleo de léxico son un indicador de la evolución de la mirada social hacia este fenómeno. Los términos sobreturismo y turismofobia tienen su génesis en el rápido desarrollo de prácticas de turismos de masas insostenibles, y las respuestas que ello ha generado (Milano, Noveli y Cheer 2019). El aumento de los flujos de viajes internacionales en todo el planeta ha llamado la atención sobre la justicia socioespacial en relación con el impacto del turismo y la gentrificación transnacional en las ciudades, ya que el crecimiento de turistas y migrantes por estilo de vida puede potencialmente reducir o incluso privar de un derecho social a la ciudad (Diaz y Jover 2021). Es por ello que el sobreturismo puede ser considerado como una de las consecuencias de estas transformaciones urbanas.

Este crecimiento del turismo, en especial en las ciudades, está siendo foco de análisis, e incluso aquellas visitadas con frecuencia están teniendo dificultades para acordar una estrategia para limitar el crecimiento del turismo (Boom et al. 2021). Estos problemas de exceso de turismo, los movimientos anti-turísticos y las externalidades negativas del turismo son conceptos clave para comprender mejor el desarrollo sostenible de los destinos turísticos (Bertochi et al. 2020). Toda esta situación crea conflicto entre los residentes locales y los visitantes, lo que hace que se requieran decisiones organizativas y de gestión competentes de diferentes niveles, con el fin de proporcionar condiciones efectivas para los turistas que sean a su vez beneficiosas para la comunidad local (Alexandrova et al. 2019b). Sin embargo, cabe también 
destacar que la falta de voluntad por parte de esta comunidad residente para aceptar más turismo no se corresponde necesariamente con percepciones negativas del mismo (Muler, Coromina y Galí 2018), ya que se considera que la percepción que tienen los residentes sobre los impactos del turismo es tanto o más que los impactos en sí mismos (Gomez y Martin 2019). En el futuro, debe cobrar importancia, como respuesta al sobreturismo, la necesidad de educar también al turista o visitante (Capocchi et al. 2019).

El impacto que el sobreturismo tiene, de forma real o percibida, en los destinos, acarrea importantes consecuencias ecológicas, sociales y culturales, conduciendo a escenarios de turismo social y ecológicamente insostenibles, volviéndose necesario que el sector se actualice o se diversifique hacia un modelo que equilibre los intereses económicos con los sociales y ecológicos. Un modelo que no dependa tanto de la llegada de una gran cantidad de visitantes o formas de turismo que traen consigo perturbaciones para la población local y el medio ambiente, sino que pueda involucrar nichos marcados por una mejor relación entre el valor agregado local y las huellas socioecológicas (Benner 2020), ya que además la sostenibilidad es uno de los principales retos de la sociedad (Cabello et al. 2021). El turismo debe continuar con su faceta más utópica de educación del ciudadano para transformar la vida de las personas y sociedades.

Una adecuada y sostenible planificación turística puede reducir considerablemente los iniciales puntos de vista negativos hacia el destino (Navarro y Capote 2020). Medir el grado de exceso de turismo es esencial para los planificadores urbanos, los administradores de ciudades y las OGD, que sienten la necesidad de acudir a indicadores exhaustivos para apoyar la toma de decisiones y la gestión del turismo en áreas urbanas congestionadas (Amore, Falk y Adie 2020). El turismo, si no se gestiona y planifica, cambia radicalmente las estructuras sociales y urbanas de una ciudad y de la vida de sus necesidades locales. Se hace por tanto fundamental que una ciudad turístico-histórica disponga de un plan estratégico que la ayude a enfrentar fácilmente problemas como el hacinamiento, las externalidades negativas y los fuertes impactos en la comunidad local (Bertocchi y Visentin 2019), abogando a un modelo turístico más sostenible. 
Esta búsqueda de políticas y sinergias de intereses entre los diferentes actores del turismo se debe convertir en una prioridad para el entorno, atendiendo siempre las necesidades y expectativas de los residentes (Fedyk et al. 2020). Además, las partes interesadas, aún teniendo algunos roles similares, tienen visiones del mundo muy diferentes, lo que puede ayudar a explicar la dificultad de cambiar el paradigma del crecimiento del turismo (Boom et al. 2021b). Se hace, por tanto, fundamental para abogar hacia un desarrollo turístico sostenible, buscar un balance entre visitantes y residentes (Cheung y Li 2019b). El éxito a largo plazo del turismo urbano sostenible requiere preservar la autenticidad de la ciudad, y hacerlo demanda intervenciones políticas basadas en una visión a largo plazo, pronóstico y participación de las partes interesadas (Boom et al. 2021c).

A esto se le añade que el decrecimiento, cuyo objetivo es unir los movimientos sociales y la investigación, ha ido surgiendo en un contexto de crisis social para hacer posible otro turismo (Milano, Novelli y Cheer 2019). Sin embargo, las estrategias basadas únicamente en el decrecimiento del turismo pueden no ser suficientes para cambiar completamente de un sector arraigado en el paradigma del "crecimiento para el desarrollo" a uno de "decrecimiento para la habilidad" (Milano, Novelli y Cheer 2019).

Los visitantes son una parte esencial en esta ecuación. Previamente a la actual pandemia causada por COVID-19, nos encontrábamos en una época de hipermovilidad en la que la propia distinción entre visitante y residente estaba en cuestión, en especial en los centros urbanos (De la Calle 2019). Por lo tanto, el sobreturismo es un fenómeno que ocurría antes del estallido de la pandemia (Montanari 2020). No obstante, las regulaciones pre pandemia permanecen, por lo que mirando al futuro, es probable que surjan con renovada intensidad situaciones y procesos de sobreturismo. Será entonces cuando las medidas de regulación urbana constituirán el instrumento básico a disposición de los gobiernos locales (De la Calle, García y Mendoza 2021), ya que a pesar de que el turismo ofrece una importante contribución económica y social al patrimonio cultural de Europa, sería perjudicial volver a los errores del pasado que llevaron al exceso de turismo (Montonari 2020).

La recuperación tras la pandemia de los espacios urbanos puede identificarse como un motivo de esperanza ambiental que simboliza la vida, la 
regeneración y la resiliencia, cuya comprensión puede contribuir al proyecto de un turismo esperanzador en la época post COVID-19 (Crossley 2020). La pandemia podría suponer un comienzo para que los gobiernos desempeñen un papel más importante y activo para mejorar la calidad de vida de los ciudadanos (Koh 2020), ya que ha hecho que se presente la urgente necesidad de configurar ciudades inclusivas y resilientes. Una de las estrategias para lograrlo es la educación ambiental, que va a permitir la concienciación y sensibilización con esta causa (Cabral y Diez 2020). Es por ello que el desafío para el sector turístico es importante, ya que la sostenibilidad indica que se han de buscar equilibrios entre medio ambiente, sociedad y economía (Romagosa 2020). El turismo sostenible ofrece, además, una mayor predisposición a la hora de cumplir los protocolos sanitarios frente al COVID-19 (Garcia y Ruz 2020), ya que responde a las necesidades de los turistas y de las regiones receptoras (Linares y Garrido 2014), además del fomento del turismo de proximidad como estrategia de reactivación, tanto para turistas como para residentes (Cabral y Diez 2020b).

Es difícil anticipar los tipos de lugares hacia los cuales los turistas serán atraídos (Judd 2013). Ante un futuro incierto, los destinos que parecen estar en una situación menos desfavorecida, más resiliente, son aquellos que tienen una oferta más diversificada, es decir, son menos dependientes de un mercado en particular (Romagosa 2020b). Nuevos paradigmas relacionados con esta diversificación son los relacionados con los Destinos Turísticos Inteligentes, cuyo nivel operativo puede abrir nuevas vías de intervención para ayudar a gestionar el sobreturismo y abogar por un modelo turístico más sostenible (Garcia, Ivars y Mendoza 2019).

Tradicionalmente, el turismo es entendido y percibido como una actividad que permite vivir experiencias diferentes de las habituales en lugares diferentes a los usuales. Sin embargo, este concepto de habitual no está automáticamente vinculado con proximidad (Diaz y Llurdés 2013). Este turismo de proximidad no es considerado sólo como estrategia de reactivación durante y tras la COVID-19, sino como una estrategia para reconvertir un destino turístico (Cabral y Díez 2020) hacia un desarrollo local (Díaz y Llurdés 2013b), ya que, además, está asociado a una práctica de turismo más sostenible (Díaz 2017), que al reducir el impacto de los transportes es más respe- 
tuoso con el medio ambiente (Astruc 2009), por lo que es entendido como una forma alternativa de entender el viaje turístico (Díaz y Llurdés 2013).

En los países desarrollados y las economías emergentes, donde se concentra la mayor parte de la demanda turística mundial y donde se espera que el turismo de proximidad ayude a salvar el sector, la situación es más prometedora que en los países en desarrollo, ya que estos últimos dependen en gran medida de los mercados emisores que provienen principalmente de países desarrollados. Es por ello que el desafío para el sector turístico mundial es importante, ya que la sostenibilidad nos dice que busquemos equilibrios, es decir, entre el medio ambiente, la sociedad y la economía (Romagosa 2020).

\section{Limitaciones del estudio y futuras líneas de investigación}

Entre las limitaciones del estudio, cabe destacar la falta de artículos que relacionan todas las palabras clave, motivo por el que se realizó una búsqueda de cada una de ellas por separado, siempre en combinación con el término turismo sostenible para establecer una relación que conduzca al logro de los objetivos marcados, mediante el uso de técnicas tanto bibliométricas como del análisis de contenido (Jurado et al. 2020).

Otra limitación deriva del propio análisis bibliomético y de contenido efectuado, pues ofrece una visión del conocimiento acumulado hasta la fecha de análisis, sin permitir extrapolaciones de los mismos.

Las futuras líneas de investigación han de ir encaminadas a completar las lagunas existentes en la literatura científica al respecto y analizar posibles estrategias viables ante la nueva situación de pandemia mundial, que conjugue el binomio ciudad y turismo bajo el objetivo de viabilidad a largo plazo. Además, la investigación ha de estar encaminada a indagar sobre las barreras psicológicas que se están creando en los turistas, en la población local y en los gobiernos, cómo inciden en los conflictos sociales y la estigmatización tras haber padecido la COVID-19 o de proceder de destinos muy castigados por dicha pandemia.

Otras posibles líneas de trabajo derivadas del análisis del contenido serían: analizar el porqué, a pesar de ser Europa el continente en el que más se dan las 
situaciones expuestas en esta investigación, es donde menos concentración de investigaciones encontramos al respecto; explorar cómo la innovación tecnológica puede apoyar la reducción de la huella de carbono en las ciudades, al ser uno de los principales retos a los que se enfrenta la sociedad (Urrutia et al. 2018); o cómo pueden contribuir a un desarrollo turístico más sostenible otras opciones alternativas, como el turismo de proximidad o turismo rural.

\section{Referencias}

Alexandrova, A.Y., Aigina, E.V., Kaverzin, I.L. y Sheresheva, M.Y. (2019). Management of overtourism problems in small historical towns. Case of Suzdal, Russia. Journal of Environmental Management \& Tourism, 10 (8 (40), 1852-1862.

Amore, A., Falk, M., y Adie, B. A. (2020). One visitor too many: Assessing the degree of overtourism in established European urban destinations. International Journal of Tourism Cities, 6 (1), 117-137.

Astruc, L. (2009). Écotourisme. Voyages écologiques et équitables. Grenoble, Glénat.

Urrutia, K., Fontan, L., Diez, F. J., Rodriguez, F. y Vicente, J. (2018). Smart Zero Carbon City Readiness Level: sistema de indicadores para el diagnóstico de las ciudades en su camino hacia la descarbonización y su aplicación en País Vasco. DYNA, 93(3), 332-338.

Benito, R.M. (2016). El turismo como sector estratégico en las etapas de crisis y desarrollo de la Economía Española. International journal of scientific management and tourism, 2(4), 81-115.

Benner, M. (2020). The decline of tourist destinations: An evolutionary perspective on overtourism. Sustainability, 12(9), 3653.

Bertocchi, D. y Visentin, F. (2019). "The overwhelmed city": Physical and social over-capacities of global tourism in Venice. Sustainability, 11(24), 6937.

Bertocchi, D., Camatti, N., Giove, S. y van der Borg, J. (2020). Venice and Overtourism: Simulating sustainable development scenarios through a tourism carrying capacity model. Sustainability, 12(2), 512.

Berry, S. \& Ladkin, A. (1997). "Sustainable tourism: A regional perspective". Tourism Management, 18(7): 433-440

Boom, S., Weijschede, J., Melissen, F., Koens, K. y Mayer, I. (2021). Identifying stakeholder perspectives and worldviews on sustainable urban tourism development using a Q-sort methodology. Current Issues in Tourism, 24(4), 520-535. 
Cabello, J.M., Navarro, E., Thiel, D., Rodríguez, B. y Ruiz, F. (2021). Assessing environmental sustainability by the double reference point methodology: the case of the provinces of Andalusia (Spain). International Journal of Sustainable Development \& World Ecology, 28(1), 4-17.

Cabral, V.N. y Diez, J. (2020). Divulgación del Proyecto Mar del Plata Natural: sostenibilidad y promoción del turismo de proximidad local. Investigación+ Acción, (23), 140-146.

Capocchi, A., Vallone, C., Pierotti, M. y Amaduzzi, A. (2019). Overtourism: A Literature Review to Assess Implications and Future Perspectives. Sustainability, 12(4), 1541.

Capocchi, A., Vallone, C., Pierotti, M. y Amaduzzi, A. (2020). Correction: Capocchi, A., et al. Overtourism: A Literature Review to Assess Implications and Future Perspectives. Sustainability 2019, 11, 3303. Sustainability, 12(4), 1541.

Capocchi, A., Vallone, C., Amaduzzi, A. y Pierotti, M. (2020). Is 'overtourism'a new issue in tourism development or just a new term for an already known phenomenon? Current Issues in Tourism, 23(18), 2235-2239.

Cazau, P. (2004). Categorización y operacionalización. Investigación Educativa Duranguense, (3), 1.

Cheung, K.S. y Li, L.H. (2019). Understanding visitor-resident relations in overtourism: Developing resilience for sustainable tourism. Journal of Sustainable Tourism, 27(8), 1197-1216.

Crossley, É. (2020). Ecological grief generates desire for environmental healing in tourism after COVID-19. Tourism Geographies, 22(3), 536-546.

De Carvalho, G.D.G., Sokulski, C.C., Da Silva, W.V., De Carvalho, H.G., De Moura, R.V., De Francisco, A.C. y Da veiga, C.P. (2020). Bibliometrics and systematic reviews: A comparison between the Proknow-C and the Methodi Ordinatio. Journal of Informetrics, 14(3), 101043.

De la Calle, M. (2019). Turistificación de centros urbanos: clarificando el debate. Boletín de la Asociación de Geógrafos Españoles, (83), $1-40$.

De la Calle, M., García, M. y Mendoza, S. (2021). Urban Planning Regulations for Tourism in the Context of Overtourism. Applications in Historic Centres. Sustainability, 13(1), 70. 
Delgado, E., Orduna, E. y Martín, A. (2018). Google Scholar as a data source for research assessment. In W. Glaenzel, H. Moed, U. Schmoch, \& M. Thelwall (Eds.), Springer Handbook of Science and Technology Indicators. Springer.

Delgado, E., Robinson, N. y Torres, D. (2014). The Google scholar experiment: How to index false papers and manipulate bibliometric indicators. Journal of the Association for Information Science and Technology, 65(3), 446-454.

Díaz, I. y Llurdés, J.C. (2013). Reflexiones sobre el turismo de proximidad como una estrategia para el desarrollo local. Cuadernos de Turismo, (32), 65-88.

Diaz, I. (2017). Being a tourist as a chosen experience in a proximity destination. Tourism Geographies, 19(1), 96-117.

Díaz, I. y Jover, J. (2021). Overtourism, place alienation and the right to the city: insights from the historic centre of Seville, Spain. Journal of Sustainable Tourism, 29(2-3), 158-175.

Duque, P. y Cervantes-Cervantes, L.S. (2019). Responsabilidad Social Universitaria: una revisión sistemática y análisis bibliométrico. Estudios Gerenciales, 35(153), 451-464.

Fedyk, W., Sołtysik, M., Olearnik, J., Barwicka, K. y Mucha, A. (2020). How Overtourism Threatens Large Urban Areas: A Case Study of the City of Wrocław, Poland. Sustainability, 12(5), 1783.

Fletcher, R., Murray, I., Blanco, A. y Blázquez, M. (2019). Tourism and degrowth: an emerging agenda for research and praxis. Journal of Sustainable Tourism, 27(12), 1745-1763.

García, M., Ivars, J. y Mendoza, S. (2019). Overtourism in urban destinations: the myth of smart solutions. Boletín de la Asociación de Geógrafos Españoles, 83(2830), 1-38.

Gómez, D. y Martín, C. (2019). Los impactos del turismo en España: diferencias entre destinos de sol y playa y destinos de interior. Cuadernos de Turismo, 43, 325-347.

Goodwin, H. (2017): “The Challenge of Overtourism”, en Responsible Tourism Partnership, octubre de 2017. Disponible en https://haroldgoodwin.info/pubs/RTP\%27WP4Overtourism01\%272017.pdf

Gössling, S. y Higham, J. (2020). The low-carbon imperative: Destination management under urgent climate change. Journal of Travel Research, 0047287520933679.

Hunter, C. (1997). Sustainable tourism as an adaptive paradigm. Annals of Tourism Research 24(4):850-867 
Jiménez, C.C., Nechar, M.C. y Vega, C.H. (2014). Sosteniendo al turismo o turismo sostenible (TS). Reflexiones Teóricas. Estudios y perspectivas en turismo, 23(2), 376-395.

Jover, J. y Díaz, I. (2020). Who is the city for? Overtourism, lifestyle migration and social sustainability. Tourism Geographies, 1-24.

Judd, D.R. (2003). El turismo urbano y la geografía de la ciudad. Revista eure, 29 (87), 51-62

Jurado, M.Á., Quintana, C. y Rodríguez, M. (2020). Trends and opportunities in research on disability and work: An interdisciplinary perspective. BRQ Business Research Quarterly, 2340944420972715.

Koens, K., Postma, A. y Papp, B. (2018). Is Overtourism Overused? Understanding the Impact of Tourism in a City Context. Sustainability, 10,4384 .

Koh, E. (2020). The end of over-tourism? Opportunities in a post-Covid-19 world. International Journal of Tourism Cities, 6, 1015-1023

Linares, H.L. y Garrido, G.M. (2014). Del desarrollo turístico sostenible al desarrollo local. Su comportamiento complejo. Pasos Revista de Turismo y patrimonio cultural, 12(2), 453-466.

Llurdés, J.C., Díaz, I. y Romagosa, F. (2014). Patrimonio minero, paisaje y turismo de proximidad: explorando sinergias. El caso de Cardona (Barcelona). In $3^{\circ}$ Crossroads of Europe y $5^{\circ}$ Congreso Europeo de Turismo Industrial, 17-20.

Manning, T., Clifford, G., Dougherty, D. y Ernst, M. (1997). Guía práctica para el desarrollo y uso de indicadores de turismo sostenible. OMT. Madrid.

Martín, A., Orduna, E., Thelwall, M. y López, E. D. (2018). Google Scholar, Web of Science, and Scopus: A systematic comparison of citations in 252 subject categories. Journal of informetrics, 12(4), 1160-1177.

Milano, C., Novelli, M. y Cheer, J.M. (2019). Overtourism and degrowth: a social movements perspective. Journal of Sustainable Tourism, 27 (12), 1857-1875.

Milano, C., Novelli, M. y Cheer, J.M. (2019). Overtourism and tourismphobia: A journey through four decades of tourism development, planning and local concerns. Tourism Planning \& Development,16(4), 353357.

Molina, N.P. (2005). ¿Qué es el estado del arte? Ciencia y Tecnología para la salud Visual y Ocular, 3(5), 73-75. 
Montanari, A. (2020). Covid-19 as an opportunity to tackle the phenomenon of overtourism in European historic centres: the case of Rome/ Covid-19 un'occasione per affrontare il fenomeno dell'overtourism nei centri storici europei: il caso di Roma. IL CAPITALE CULTURALE. Studies on the Value of Cultural Heritage, (11), 285-305.

Muler, V., Coromina, L. y Galí, N. (2018). Overtourism: residents' perceptions of tourism impact as an indicator of resident social carrying capacity-case study of a Spanish heritage town. Tourism Review, 73 (3), 277-296.

Navarro, F.A. y Capote, A.L. (2020). ¿Overtourism en la ciudad de Granada?: una aproximación a la percepción de turistas, residentes y partidos políticos locales. Cuadernos geográficos de la Universidad de Granada, 60(1), 35-53.

Navarro, E., Ortega, G. y Torres, E. (2020). Propuestas de reflexión desde el turismo frente al COVID-19. Incertidumbre, impacto y recuperación. Instituto Universitario de Investigación de Inteligencia e Innovación Turística de la Universidad de Málaga: Málaga, 2020.

Pimentel de Oliveira, D. (2020). La sostenibilidad como apoyo en la desescalada de la COVID-19: una comparación al modelo de Doxey. In: M. Simancas Cruz, R. Harnández Martín and N. Padrón Fumero, ed., Turismo pos-COVID-19. Reflexiones, retos y oportunidades., 1st ed. La Laguna: Cátedra de Turismo Caja Canarias-Ashotel de la Universidad de La Laguna, 271-280.

Pons, G.X., Blanco-Romero, A., Navalón-García, R., Troitiño-Torralba, L. y Blázquez-Salom, M. (2020). Sostenibilidad Turística: overtourism vs undertourism. Mon. Soc. Hist. Nat. Balears, 31, 610.

Rädiker, S. y Kuckartz, U. (2021). Análisis de Datos Cualitativos con MAXQDA: Texto, Audio, Video. BoD-Books on Demand.

Ravindran, V. y Shankar, S. (2015). Systematic reviews and meta-analysis demystified. Indian journal of rheumatology, 10(2), 89-94.

Rivera, J. y Pastor, R. (2020). ¿Hacia un turismo más sostenible tras el covid-19? Percepción de las agencias de viajes españolas. Gran tour, revista de investigaciones turísticas, 21. Recuperado a partir de http://eutm.es/grantour/index.php/grantour/article/view/153

Romagosa, F. (2020). The COVID-19 crisis: Opportunities for sustainable and proximity tourism. Tourism Geographies, 22(3), 690-694.

Sgier, L. (2012). Qualitative data analysis. An Initiat. Gebert Ruf Stift, 19, $19-21$. 
Swarbrooke, J. (1998). Sustainable tourism management. CABI Publishing, New York

Valero Escandell, J.R. y García-Tortosa, F. (2020). Overtourism urbano y cambios demográficos. En Sostenibilidad turística:" overtourism vs undertourism" (519-530). Societat d'Història Natural de les Balears.

Vargas, G. y Calvo, G. (1987). Seis modelos alternativos de investigación documental para el desarrollo de la práctica universitaria en educación. Revista Educación superior y desarrollo, 5(3), 7-37.

Vargas, M.G., Higuita, C.G. y Muñoz, D.A.J. (2015). El estado del arte: una metodología de investigación. Revista Colombiana de Ciencias Sociales, 6(2), 423-442.

Vieira, E.L., Da Costa, S.E.G., De Lima, E.P. y Ferreira, C.C. (2019). Application of the Proknow-C methodology in the search of literature on performance indicators for energy management in manufacturing and industry 4.0, Procedia Manufacturing, 39, 1259-1269.

Zenker, S. y Kock, F. (2020). The coronavirus pandemic-A critical discussion of a tourism research agenda. Tourism management, 81, 104164. 
